\title{
UV Wind Variability in B Supergiants and its Implications for Wind Structures
}

\author{
Derck Massa $^{1}$ and Raman K. Prinja ${ }^{2}$ \\ 1 Raytheon STX, NASA/GSFC, Code 631.0, Greenbelt, MD 20771, USA \\ 2 Astronomy Department, University College London, Gower Street, London WC1E \\ 6BT, England, U.K.
}

\begin{abstract}
We discuss why B supergiant winds are particularly well suited for wind studies, and present or refer to dynamic spectra which suggest the presence of disks, bifurcated winds, shock formation, rotationally modulated winds and the spontaneous generation of wind enhancements. They underscore the strength and richness of wind variability in B supergiants and the challenges these phenomena present to theoretical studies of stellar winds.
\end{abstract}

\section{Why BIs are well suited for wind studies}

B supergiant (BI) winds are ideal for studying wind variability because: 1) their wind lines are often well-developed but unsaturated; 2) their wind lines cover a range of ionization, close to the dominant stage of the wind (often $\mathrm{C}$ II $-\mathrm{N} \mathrm{V}$, and Si III \& IV); 3) their winds are less chaotic than O stars, making coherent features easier to identify and track; 4) their winds have lower terminal velocities, so individual structures evolve more slowly and the $\mathrm{Si}$ IV $\lambda \lambda 1400$ doublets are decoupled; 5) their strong UV photospheric lines are excellent $T_{\text {eff }}$ - luminosity diagnostics, and 6) the strongest UV photospheric lines sample the wind-photosphere interface, often displaying wind behavior.

\section{The observations}

Over its lifetime, IUE obtained 15 good time series of $10 \mathrm{BIs}$, with time coverages of $\sim 1-30$ days, and sampling rates from $30 \mathrm{~min}$. to once a day. Four of these have been described in detail by Massa et al. (1995 - evidence for $v=0$ structure), Prinja et al. (1995), Fullerton et al. (1997 - evidence for Co-Rotating Interaction Regions), Howarth et al. (1998), Prinja et al. 1997 a 2-component wind and disk structure), Massa et al. (1998, 1999 - evidence for a photospheric - wind connection). Prinja et al. (1999) will provide a complete overview of all these series. Here we demonstrate a few common aspects of them and concentrate on ionization structure.

UV wind line variability in BIs can imply line of sight optical depth variations up to 10 over localized wavelengths and a factor of 2 or more integrated 

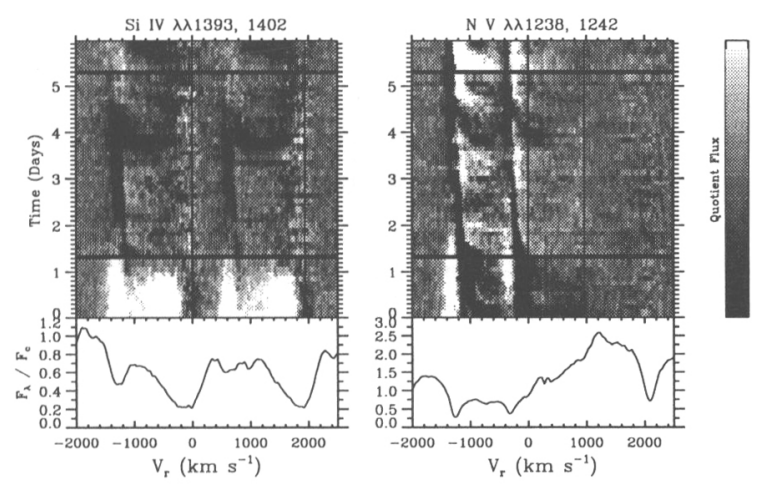

Fig. 1. Dynamic spectra of a 1993 time series of HD 150168. This star is an SB1 binary, and the individual spectra have been aligned on the photospheric velocity of the primary. The spectra are normalized be the series mean (shown at the bottom of each panel), and the extremes of the scale run from $0.75-1.25$.
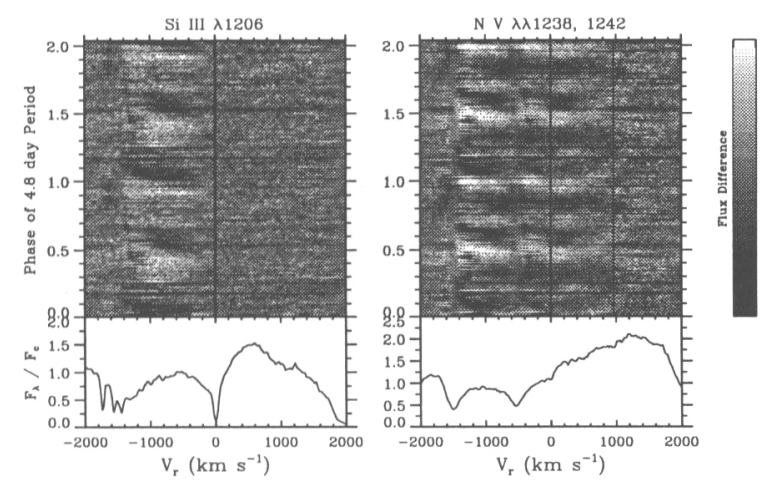

Fig. 2. Dynamic spectrum of 1995 ("MEGA") data for HD 64760 . The 16 days of spectra are arranged relative to the 4.8 day period determined by Fullerton et al. (1997). The spectra are differences from the series mean spectrum.

over the entire profile. These variations can occur as normal DACs, which appear at intermediate $v$ and narrow as they propagate to higher $v$; bowed absorptions, which appear at intermediate velocity and propagate blueward and redward simultaneously and are thought to be related to CIRs; and other absorptions, which often simply appear over a large range of velocity all at once. In addition, the features often extend to $v=0$ suggesting an origin on 
stellar surface and, when the extent of the series is adequate, often recur, usually on the rotation timescale - also suggesting surface features. Finally, when lines from different ions are compared, we see ionization stratification, which means that related structures occur at different velocities in different ions - often just an optical depth effect, and ionization variability which implies that the ionization state of a specific feature changes with time.

All of the previous effects can be seen in the accompanying figures. Figure 1 of the B1 Iab-Ib star HD 150168. Near the beginning of the series, there is a maximum in $\mathrm{N} \mathrm{V}$ which correponds to a minimum in Si IV - ionization variability. Near day 4 , a bowed structure shows appears with its minimum in $\mathrm{N} V$ lagging the Si IV minimum - ionization stratification. Figure 2 is for HD 64760, "phased" on a 4.8 day period. Notice that the $\mathrm{N} v$ data appear to have nearly twice the frequency of the Si IV data - ionization variability.

\section{Conclusions}

BI wind activity is rich and varied, with similar structures occurring in very different stars and different structures occurring in same star at different times. The variability evolves smoothly, and several lines of evidence point to surface features as the origin of much of the activity, although some features are difficult to reconcile with this interpretation. We have also detected photospheric line variability which is only sometimes associated with variability in the the wind lines.

\section{References}

Fullerton, A.W, Massa, D., Prinja, R.K., Owocki, \& Cranmer 1997, A\&A 327, 699 Howarth, I.D., et al. 1998, MNRAS 296, 949

Massa, D., Fullerton, A.W., \& Prinja, R.K. 1995, ApJ 452, 842

Massa, D., Fullerton, A.W., \& Prinja, R.K. 1998, in ESO Workshop on Cyclical Variability in Stellar Winds, p121

Massa, D., Prinja, R.K., Fullerton, Howarth, \& Owocki 1999, in preparation

Prinja, R.K., Massa, D., \& Fullerton, A.W. 1995 ApJ 452, L61

Prinja, R.K., Massa, Fullerton, Howarth, \& Pontefract 1997, A\&A 318, 157

Prinja, R.K., Massa, D., \& Fullerton, A.W. 1999, in preparation

\section{Discussion}

A. Kaufer: Do you see any relation between the "bananas" and the much more slowly evolving DACs in the CIR picture?

D. Massa: The DACs seem to recur less frequently, if at all; and we just don't have runs long enough to determine whether they are periodic. 
H. Henrichs: Have you checked whether simultaneous photometry from HIPPARCOS is available?

D. Massa: No.

S. Shore: For the spontaneous (that is, the non-propagating) features, is there anything like a characteristic velocity at which they appear?

D. Massa: This is somewhat tied up with the definition, in that these features are typically identified at intermediate velocity. However, they can appear over a wide range of velocities reaching to $v=0$ at some times.

J. Dachs: Why do you suppose $\gamma$ Arae to show a disk in its wind?

D. Massa: It seems to have two separate winds: one has a terminal velocity of about $-750 \mathrm{~km} / \mathrm{s}$ in Si III and Si IV, and another is seen in the edge of $\mathrm{N} \mathrm{V}$ with a terminal velocity of about $-1200 \mathrm{~km} / \mathrm{s}$. Also, the Si IV profiles look like those in HD 93521: flat-bottomed with little emission. These are indicative of a disk. Conversely, the $\mathrm{N} v$ profiles have strong emission and weak absorption, which is indicative of a hot wind confined to a cone above the poles.

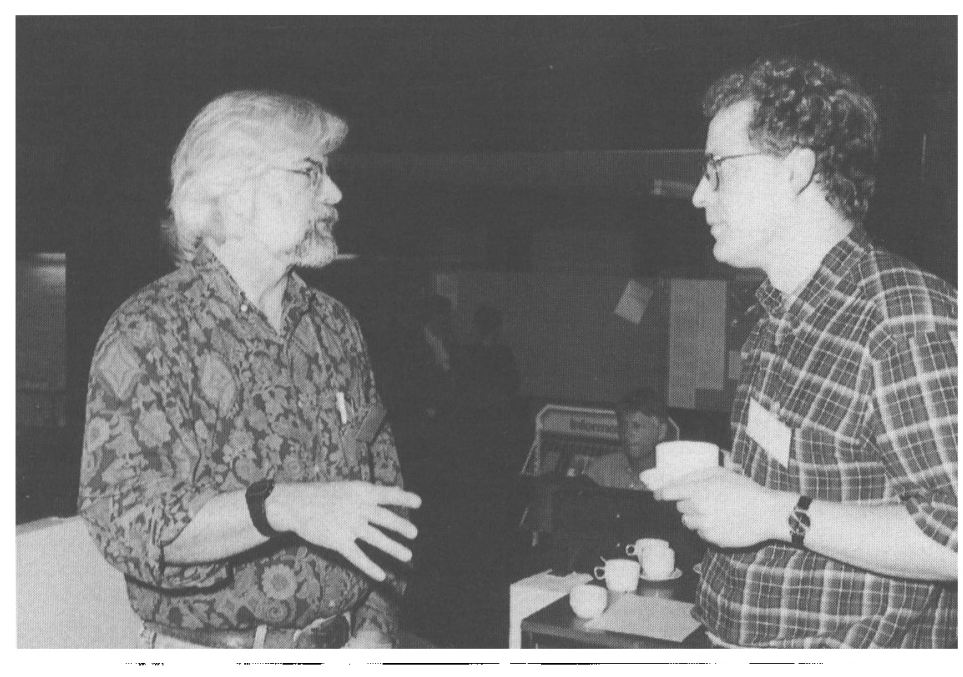

Derck Massa and Alexander Fullerton 